\title{
34. Membrane Elevation of Sea-urchin Egg by Intracellular Injection*.
}

\author{
By Takeo Kamada. \\ Zoological Institute, Faculty of Science, Tokyo Imperial University. \\ (Comm. by N. YATSU, M.I.A., May 12, 1941.)
}

Various kinds of solutions were injected into the unfertilized egg of Strongylocentrotus pulcherrimus, with a view to examining the direct effect of these substances on the process of membrane elevation. The technique of injection was fundamentally not very much different from that adopted in the injection experiment with Paramecium (Kamada, Proc. 14, 260). The tip of the micropipette was always $2 \sim 3$ microns in external diameter. The animals were brought from the Misaki Marine Biological Station and preserved in a refrigerator.

Like the eggs of many other forms, the unfertilized egg of this material is provided with a thin hyaline shell over the real cell membrane. If the injection is made with a micropipette barely inserted into the peripheral layer of the egg, the solution can be discharged in the interstice between these two membranes, thus forming a hyaline space there and causing an inflation of the outer shell which has hardly been visible before. The outer shell is highly elastic, and by the injection it can be distended so greatly as to push aside the neighboring eggs. When the injection is stopped, the injected solution is driven out completely by the tension of the outer shell and the hyaline space vanishes again. If, however, a colloidal solution (e. g., hen's egg white, diluted by half with $5 / 4 \mathrm{M} \mathrm{NaCl}$ and then filtered) is injected instead, the space does not disappear, though much reduced in size, after the injection. Therefore it is highly probable that the outer shell is a dialyzing membrane. On the other hand, reagents, which would cause the membrane elevation when injected intracellularly, are introduced in this space without effect. This induces us to believe that, inside the space, there exists the plasma membrane which checks the free pass of solutes. Therefore it may be suggested that, on stimulation, if some substance of colloidal size is discharged by the cell into the space, or more reasonably, if the cell secretes in the space some substance of molecular size which can greatly increase the degree of dispersion of the colloidal aggregates already existing there and adhering to the plasma membrane, the outer shell will balloon up by taking sea-water colloidosmotically from without. The membrane elevation described in this paper is an actual separation of an outer hyaline membrane from an inner membrane, which can be observed at the cell cortex immediately after an injection of suitable substance in the cytoplasm. The hyaline space develops by an elevation of the outer membrane (probably owing

\footnotetext{
* Aided by the Governmental Research Fund for Science from the Department of Education.
} 
to the colloid osmosis explained above) and not by the shrinkage of the cytoplasm. However, whether or not these separated membranes are identical with the two membranes discussed above, can not as yet be made out with certainty. Cytolysis, usually not accompanied by bursting, causes the swelling of the egg and this seems to suggest that as the plasma membrane is destroyed, the cytoplasmic colloids are brought in a direct contact with the outer dialyzing membrane. It was often observed after cytolysis, that the entire cell granules form an aggregated mass within the hyaline envelope in the course of several minutes, so that a more or less transparent space appears secondarily at the periphery. This transparent space should not be confused with that due to the membrane elevation, because the latter becomes visible within a few seconds subsequent to the so-called "roughening response" and without any visible destruction of the plasma membrane.

The sign of what we call successful injection is the scattering of cell granules observed at the moment of injection around the inserted tip of micropipette, provided that the injection is made with the least possible mechanical injury due to the insertion of the pipette. However, even though an effective reagent is used and the injection is successful in this sense, if too little solution is injected, no membrane elevation takes place, while too much solution causes cytolysis without the cortical response. Therefore, the membrane-elevating capacity of reagents should be examined at their optimal dose. However, the range of the optimal dose is rather wide in general. Moreover, the membrane elevation induced by injection is not a reaction of all-or-none type (e. g., only a small blister appears on the egg surface when a small dose is injected). Therefore, to find out the optimal dose for each reagent, we can rather easily resort to the trial-and-error method. However, there are many reagents which induce no reaction with a small dose of injection but have only cytolytic effects on the egg with a dose above a threshold level. These reagents are labelled in my list to be incapable of inducing the membrane elevation at least under the given condition.

The effective reagents so far obtained were in general toxic to the egg when injected. Although the membrane elevated by injection could not be distinguished in appearance from that of the normal fertilization, yet the cell underwent disintegration, the contour having become ill defined usually within 2 10 minutes. In some rather exceptional cases, eggs manifested no sign of disintegration for about 3 hours after the membrane elevation being induced in sea-water by the injection of $0.06 \mathrm{M} \mathrm{K}$ citrate- $0.5 \mathrm{M} \mathrm{KCl}(0.06 \mathrm{M} \mathrm{K}$ citrate made up with $0.5 \mathrm{M} \mathrm{KCl})$, but they showed neither the cleavage furrow nor the astral figures so long as my observation lasted (room temperature $13^{\circ} \mathrm{C}$ ).

In order to bring about the cortical response, there seems to be a definite relation between the nature of solution to be injected and that of the medium in which the egg is suspended, as is given in the accompanying table (the plus sign means membrane elevation, and the minus sign non-elevation). 


\begin{tabular}{|c|c|c|c|c|c|c|c|}
\hline \multirow{2}{*}{ SOLUTION INJECTED } & \multicolumn{7}{|c|}{ EXTERNAL MEDIUM* } \\
\hline & A & $\mathrm{B}$ & $\mathrm{C}$ & $\mathrm{D}$ & $\mathrm{E}$ & $\mathrm{F}$ & G \\
\hline $0.012 \mathrm{M}$ butyric acid- $0.5 \mathrm{M} \mathrm{NaCl}$. & & & & + & & & \\
\hline $0.05 \mathrm{M}$ oxalic acid- $0.5 \mathrm{M} \mathrm{NaCl} \ldots$ & & & & + & & & \\
\hline $0.05 \mathrm{M} \mathrm{Na}$ oxalate- $0.5 \mathrm{M} \mathrm{NaCl}$ (non-buffered) & & & & + & & & \\
\hline $0.05 \mathrm{M} \mathrm{Na}$ oxalate- $0.5 \mathrm{M} \mathrm{NaCl}(\mathrm{pH}: 4.6 \sim 4.8)$ & \pm & + & + & + & + & + & + \\
\hline $0.06 \mathrm{M} \mathrm{K}$ citrate- $0.5 \mathrm{M} \mathrm{KCl}$ & \pm & + & + & + & + & + & + \\
\hline distilled water $\ldots$ & - & - & - & - & + & + & + \\
\hline $0.5 \mathrm{M} \mathrm{KCl} \ldots .$. & - & - & - & - & + & + & + \\
\hline $0.5 \mathrm{M} \mathrm{NaCl} \ldots \ldots . .$. & - & - & - & - & + & + & + \\
\hline $0.33 \mathrm{M} \mathrm{CaCl}_{2} \ldots \ldots \ldots \ldots$ & - & - & - & - & - & + & + \\
\hline sea-water (natural) & & & & - & & & \\
\hline paraffin oil ........... & & & & - & & & \\
\hline $1 \mathrm{M}$ glucose ... & & & & - & & & \\
\hline $1 \mathrm{M}$ urea & & & & - & & & \\
\hline Control (microneedle, tip : 2 3 microns) & - & - & - & - & + & + & + \\
\hline
\end{tabular}

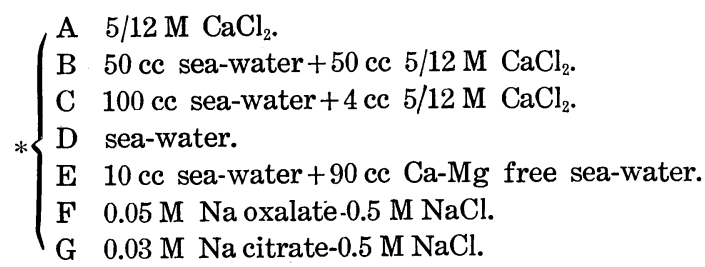

The results of experiment may be described as follows:

1) $\mathrm{K}$ citrate and $\mathrm{Na}$ oxalate possess a somewhat unique position in the table. Injection of these solutions causes the membrane elevation irrespective of the nature of external media so far investigated. In pure $\mathrm{CaCl}_{2}$ solution, a slight but unmistakable membrane elevation was observed, but within about 15 minutes of immersion the injection usually became ineffective. This change is reversible and, upon quick return to sea-water, the membrane could be lifted by injection of these salts as before. In some batches of the egg the response could not be obtained, owing probably to the situation that the inhibitory effect of the external Ca-ions had established too soon.

The $\mathrm{pH}$ value of $\mathrm{Na}$ oxalate solution was shifted to $4.6 \sim 4.8$, without modifying the concentration of oxalate anion, by diluting it with oxalic acid of the equivalent concentration, and the effect of injection of oxalate solution was found to be independent of its $\mathrm{pH}$ value at least qualitatively.

That the effect is not attributable to the cation ( $\mathrm{Na}$ or $\mathrm{K}$ ) of these solutions, is clear from the results obtained with $\mathrm{NaCl}$ or $\mathrm{KCl}$. On this account, it may be suggested that the diminution of free $\mathrm{Ca}$ ions within the egg starts a chain of events which is followed by the cortical response.

2) The injection of Ca-ions has no effect, despite that no visible boundary is formed around the injected drop and the solution seems to mix with the protoplasm more or less freely. However, in Ca-free media, 
the injection of Ca-ions also produced the membrane elevation. This seems to confirm Heilbrunn's hypothesis that the first stage of egg activation is the removal of Ca-ions from the cell cortex (which might be accomplished in the present case by placing the egg in Ca-free media) and the second stage is the increase in Ca-ion concentration at the cell interior (which may be effected in this case by the injection of Ca-ions) (cf. Heilbrunn: An outline of general physiology, Chapter 37 1937). However, such an explanation is by no means valid to the result obtained with citrate or oxalate anions mentioned before. It is important to remark here that, in order to induce the cortical response by injection of Ca-ions, the amount of the injected solution must be very little, otherwise no membrane elevation takes place. Furthermore, if the mechanical treatment at the time of insertion of the micropipette was imitated with a microneedle of equal diameter, it was found that the simple insertion of the micropipette is enough for the establishment of membrane elevation in a medium relatively poor in Ca-ions. It is therefore rather reasonable to consider that the mechanical stimulation somehow increases the permeability of cell membrane and the exchange of intracellular $\mathrm{Ca}$-ions with external Na-ions is newly enhanced across the cell membrane. Consequently the free Ca-ions within the cell are so diminished as to induce the membrane elevation. In the Ca-free media the loss of intracellular Ca-ions due to the ionic exchange may probably exceed the gain brought about by the injection of the Ca-ions, while in the medium $\mathrm{E}$ of the table the former may not be so marked as to cancel the latter.

3) In the media of comparatively low Ca-ion concentration, injection of various solutions, even of distilled water, becomes effective in causing the membrane elevation: This may be attributed to the mechanical stimulation of the micropipette as was stated before. It seems, however, possible that the injection of solutions other than $\mathrm{CaCl}_{2}$ might result in dilution of the cytoplasmic fluid and consequently in reduction of the intracellular Ca-ion concentration. As a matter of fact, if the eggs (adapted in the medium $\mathrm{C}, \mathrm{D}$ and $\mathrm{E}$ given in the table) were put into distilled water, the egg enormously swelled but, just before the cytolysis, the cortical response appeared, owing probably to the dilution of cytoplasmic fluid with the water osmotically absorbed. However, if the injected amount of solution be taken into consideration, the diluting effect of injection, if any, seems to be of a minor factor. In the media rich in Ca-ions, all injections except that of citrate or oxalate anions are ineffective, suggesting that the simple dilution of intracellular Casalts with the usual amount of injected solutions is not large enough, especially under such a condition that the cell might gain Ca-ions by the ionic exchange across the cell membrane.

As to what happens within the cell in association with the diminution of intracellular Ca-ions, no adequate explanation is at hand, but the postulation proposed in connection with the ciliary reversal of Paramecium which is also induced by reduction of intracellular Ca-ions, seems to be applicable to the present case (cf. Kamada, Proc. 16, 241).

It may be added here that, if it be true that butyrate anions form 
more or less undissociated molecules with Ca-ions within the cytoplasm, the membrane elevation caused by butyric acid may also belong to the same category of phenomenon as here considered.

4) As has been remarked, citrate or oxalate anions were injected often without effect into the egg which had been immersed in $5 / 12 \mathrm{M} \mathrm{CaCl}_{2}$ solution. According to the explanation given above, it may be said that the external Ca-ions penetrate into the cell and, in order to remove these excess free Ca-ions, some more citrate or oxalate anions are needed, but, owing to the increased toxicity of injected anions, the egg is killed before the onset of the cortical response.

It may also be considered that the fertilization membrane is hardened, and hence becomes less distensible, by Ca-ions (cf. Sugiyama, Journ. Fac. Sci. Imp. Univ. Tokyo, Zool. 4, 501), since, if the eggs treated with butyric acid (2.4 cc $\mathrm{M} / 2$ butyric acid +100 cc sea-water) for 30 seconds were transferred to modified sea-water containing various amounts of Ca-ions, the resulting membrane elevation was dependent on the Ca-ion concentration of the second solution. In fact, by employing $5 / 12 \mathrm{M} \mathrm{CaCl}_{2}$ as the second solution, the membrane elevation could be inhibited. However, if the egg thus showing no membrane elevation in pure $\mathrm{CaCl}_{2}$ solution was then put into a hypertonic solution $\left(5 / 12 \mathrm{M} \mathrm{CaCl}_{2}-5 / 8 \mathrm{M} \mathrm{NaCl}\right)$, the whole egg shrank with a wrinkled surface, leaving no smooth hyaline membrane (fertilization membrane) separated outside. Therefore it is rather difficult to consider that the elastic tension of fertilization membrane is enhanced in pure $\mathrm{CaCl}_{2}$ solution so as to prevent mechanically the membrane from being distended. It is likely that, in this case, the real separation of the fertilization membrane from the plasma membrane is inhibited by Ca-ions. On the other hand, if the eggs treated with butyric acid were placed in $1 \mathrm{M}$ glucose, the cortical response could be observed. Hence it seems probable that the role played by Ca-ions in external media is, at least in part, to make the colloidal aggregates osmotically less active (by decreasing the degree of dispersion) in the space between the fertilization membrane and the plasma membrane, and in a strong $\mathrm{CaCl}_{2}$ solution the two membranes may be glued together, so to speak, by a coagulated cement.

5) The eggs, even after 24 hours' standing in $0.05 \mathrm{M}$ Na oxalate$0.5 \mathrm{M} \mathrm{NaCl}$ (kept in a refrigerator), showed still the cortical response, if tested by injection of $0.06 \mathrm{M} \mathrm{K}$ citrate- $0.5 \mathrm{M} \mathrm{KCl}$ or of $0.33 \mathrm{M} \mathrm{CaCl}_{2}$ as well as by mechanical stimulation with the microneedle. This stands in a striking contrast with the case of Arbacia egg, which, according to Moser (Journ. Exp. Zool., 80, 455), becomes insensitive to the pricking stimulation for membrane elevation soon (2 minutes ?) after immersion in ammonium oxalate sea-water.

It is interesting to note that, in the present material, the egg, if placed in Ca-free media, becomes gradually susceptible to the injection of citrate or oxalate anions, i. e., when citrate or oxalate anions were injected, although some eggs showed the nicely elevated membrane, other eggs in one and the same preparation were killed without an indication of cortical response. Thus the high percentage of membrane 
elevation became difficult to be obtained by injection of citrate or oxalate anions after a prolonged immersion of the egg in Ca-free media.

After about 40 hours' immersion of the egg in $0.05 \mathrm{M}$ Na oxalate$0.5 \mathrm{M} \mathrm{NaCl}$ (kept in a refrigerator), the cortical response to stimulation by means of the above three methods was no longer demonstrable. However, this change is reversible, upon return to sea-water the egg having regained its capacity to respond with membrane elevation to the injection of $0.06 \mathrm{M} \mathrm{K}$ citrate- $0.5 \mathrm{M} \mathrm{KCl}$. It may therefore be conceivable that in Ca-free media the Ca-ions are lost only very slowly from the cell by an ionic exchange across the cell membrane, so that, in the long run, there would be too little amount of residual Ca-ions within the cell to be removed effectively for the initiation of the cortical response. It may further be remarked that, if the rate of diminution of intracellular Ca-ions be less than a certian value, the cortical response will not be induced in spite of a considerable loss of intracellular Ca-ions.

6) When the external. medium of the egg was carefully replaced by $1 \mathrm{M}$ glucose solution, the membrane elevation appeared. However, $1 \mathrm{M}$ glucose, when injected into the egg, could not induce the membrane elevation. It is rather interesting to find that a reagent is effective when applied externally and otherwise when introduced internally. It may be suggested here that glucose which can pass through the outer dialyzing membrane, acts directly (without entering the cell proper) on the colloidal aggregates adhering to the outer surface of the plasma membrane and makes them colloidosmotically active so as to pull the external solution into the space beneath the dialyzing membrane.

Urea is a parthenogenetic agent for the present material when applied externally (Motomura, Sci. Rep. Tohoku Imp. Univ. Biol. 9, 33), whereas $1 \mathrm{M}$ urea injected has no effect at all. Thus, as to the mode of action, urea seems to belong to the same category of reagents as glucose.

\section{Summary.}

Injection of various solutions into the unfertilized egg of a seaurchin, Strongylocentrotus pulcherrimus, shows that the membrane elevation occurs when the concentration of intracellular free Ca-ions is suddenly reduced. In a medium relatively poor in Ca-ions, mechanical stimulation due to insertion of the micropipette seems to induce the diminution of intracellular free Ca-ions, probably by an enhanced ionic exchange across the cell membrane. A prolonged subjection of the egg to Ca-free media makes the cell insensitive to the stimulation for membrane elevation, but this change is reversible.

A dialyzing membrane which covers the plasma membrane of the unfertilized egg, is demonstrated by injection of a colloidal solution in the interstice between these two membranes. A possibility is pointed out that the diminution of intracellular $\mathrm{Ca}$-ions may initiate a chain of events which make the egg to secrete in the interstice some substance so as to increase greatly the degree of dispersion of colloidal 
aggregates already existing in the interstice and to induce a colloidosmotic swelling in the interstice. It is suggested that some parthenogenetic substance may act directly on the colloidal aggregates in the interstice, without entering the cell and hence without reducing the intracellular Ca-ion concentration.

I am grateful to Mr. Takayuki Tanaka for his kindness in supplying me with the sea-urchins for the present work. 